\title{
A CUBIC COUNTERPART OF JACOBI'S IDENTITY AND THE AGM
}

\author{
J. M. BORWEIN AND P. B. BORWEIN
}

ABSTRACT. We produce exact cubic analogues of Jacobi's celebrated theta function identity and of the arithmetic-geometric mean iteration of Gauss and Legendre. The iteration in question is

$$
a_{n+1}:=\frac{a_{n}+2 b_{n}}{3} \text { and } b_{n+1}:=\sqrt[3]{b_{n}\left(\frac{a_{n}^{2}+a_{n} b_{n}+b_{n}^{2}}{3}\right)} .
$$

The limit of this iteration is identified in terms of the hypergeometric function ${ }_{2} F_{1}(1 / 3,2 / 3 ; 1 ; \cdot)$, which supports a particularly simple cubic transformation.

\section{INTRODUCTION}

The crux of this paper is a surprisingly simple cubic analogue of the arithmetic-geometric mean iteration of Gauss and Legendre and is given in Theorem 2.1(b) and Theorem 2.3(a). The arithmetic-geometric mean iteration lies at the heart of elliptic function and theta function theory [4]. In Chapter 21 of Ramanujan's second notebook [3] and in his 1914 paper on $\pi$ [9], Ramanujan indicates the existence of hypergeometric functions that admit transformations and modular equations similar to those possessed by the complete elliptic integral of the first kind, $K$, which in hypergeometric terms is $(\pi / 2){ }_{2} F_{1}\left(1 / 2,1 / 2 ; 1 ; k^{2}\right)$. This similar behaviour occurs for the function ${ }_{2} F_{1}\left(s+1 / 2,-s+1 / 2 ; 1 ; k^{2}\right)$, when $s:=1 / 3,1 / 4,1 / 6$, and essentially for no other hypergeometric functions.

For $s:=1 / 3,1 / 4$, the simple explicit algebraic relationship between $K$ and the corresponding hypergeometric function is given in $\S 5.5$ of [4], wherein, for $s:=1 / 6$, a less simple relationship is also given. These considerations lead to what Ramanujan calls "corresponding theories" [8,9] of elliptic functions based on ${ }_{2} F_{1}\left(s+1 / 2,-s+1 / 2 ; 1 ; k^{2}\right)$, when $s:=1 / 3,1 / 4,1 / 6$, and to his many remarkable series for $1 / \pi$, including that used by Gosper (see $\S 5.5$ of

Received by the editors January 22, 1989.

1980 Mathematics Subject Classification (1985 Revision). Primary 33A25; Secondary 33A30, $11 \mathrm{~F} 10,39 \mathrm{~B} 10$.

Key words and phrases. Mean iterations, theta functions, hypergeometric functions, generalised elliptic functions, cubic transformations, pi, Ramanujan.

Research partially supported by the Natural Sciences and Engineering Research Council of Canada. 
[4]) and extending to the series used by the Chudnovskys in recent large scale computations of pi (see [7]).

In this paper we primarily analyse the case $s:=1 / 6$, which gives rise to a correspondingly simple cubic transformation of ${ }_{2} F_{1}(1 / 3,2 / 3 ; 1 ;)$. The outline of this work is as follows. We first introduce a general parametric family of iterations $(N>1)$ that specialize to the classical AGM $(N=2)$ and to our iteration for $(N=3)$. We then indicate the preferred role of these two parameters; establish the underlying cubic transformation possessed by ${ }_{2} F_{1}(1 / 3,2 / 3 ; 1 ;)$; provide identification (uniformization) of ${ }_{2} F_{1}(1 / 3,2 / 3 ; 1 ;)$ and of the cubic transformation in terms of both one-dimensional and two-dimensional theta functions, and list some related identities including Lambert series. We also include its precise logarithmic asymptotic behaviour. We briefly consider the corresponding results for $s:=1 / 4$. (For $s:=1 / 3$ everything reduces to a study of Klein's absolute invariant $[4,10]$ as follows from Proposition 5.7 in [4]; this also leads to the Chudnovskys' series [7,6].) Finally, we.write down three very simple iterations for $\pi$ (one quadratic, one cubic, and one quartic) which obtain from the previous analysis.

As much as possible our notation and terminology are as in Whittaker and Watson [11] and in [4]. Some arguments are similar to ones detailed in [4] and these will be given fairly briefly. In addition, we list a few extra verifiable assertions whose derivations will be detailed in [5]. Those familiar with the classical AGM will observe that to an uncanny degree our results are what obtains if "twos" are replaced by "threes."

\section{A general iteration}

Let $N>1$ be given. Consider the two term (mean) iteration $A G_{N}$ which begins with $a \geq b>0$, sets $a_{0}:=a$ and $b_{0}:=b$, and computes

$$
a_{n+1}:=\frac{a_{n}+(N-1) b_{n}}{N} \text { and } c_{n+1}:=\frac{a_{n}-b_{n}}{N} \text {, }
$$

where

$$
b_{n}^{N}:=a_{n}^{N}-c_{n}^{N}
$$

Then the values $a_{n}$ (and $b_{n}$ ) converge to a limit, $A G_{N}(a, b)$, with order $N$, essentially because

$$
a_{n+1}^{N}-b_{n+1}^{N}=\left(\frac{a_{n}-b_{n}}{N}\right)^{N} \text {. }
$$

If (suppressing $N$ ) we introduce the involution

$$
x^{*}:=\sqrt[v]{1-x^{N}}
$$

we may observe that the ratio $k_{n}:=c_{n} / a_{n}$ is computable from

$$
k_{n+1}=\frac{1-k_{n}^{*}}{1+(N-1) k_{n}^{*}} \text {. }
$$


Also, the ratio $R_{N}(x):=A G_{N}\left(1, x^{*}\right) / A G_{N}(1, x)$ satisfies the functional equation:

$$
R_{N}(y)=N R_{N}(x)
$$

when

$$
y=\frac{1-x^{*}}{1+(N-1) x^{*}} .
$$

This much is true for any $N$ : to analyse $A G_{N}(1, x)$ further it is relevant to ask if $R_{N}(x)$ is asymptotic to a power of a logarithm as $x \rightarrow 0$. This, given the superiniear convergence of the underlying iteration, can be numerically checked and one discovers that such appears to be the case for $N:=2,3$ and for no other small integer or reasonable fraction. Given the existence of a logarithmic asymptote, one knows that $A G_{N}(1, x)$ must be expressible in terms of elliptic functions; as is classical for $N=2$ and as we now make explicit for $N=3$.

In fact, this is not the route we initially followed in discovering these results. We began with Ramanujan's quadratic modular equation listed in Remark 2.4(i) below and working by analogy with the AGM we symbolically discovered (in MAPLE) Theorem 2.3(a) and then Theorem 2.1(b). At some level this was a proof of the results, given the modular underpinnings as described in the proof of Theorem 2.3. We have, however, indicated more conventional proofs. These are still verificational and simple direct proofs would be much more satisfactory.

\section{THE QUADRATIC AND CUBIC ITERATIONS}

In the case of $A G_{2}$ we begin with $a_{0}:=a, b_{0}:=b$ and compute

$$
a_{n+1}:=\frac{a_{n}+b_{n}}{2} \text { and } b_{n+1}:=\sqrt{a_{n} b_{n}},
$$

while in the case of $A G_{3}$ we compute

$$
a_{n+1}:=\frac{a_{n}+2 b_{n}}{3} \text { and } b_{n+1}:=\sqrt[3]{b_{n}\left(\frac{a_{n}^{2}+a_{n} b_{n}+b_{n}^{2}}{3}\right)} .
$$

What is remarkable about these two iterations is that they both have limits easily expressible in terms of familiar nonalgebraic special functions. The former is classical and well appreciated; the latter appears both new and unlikely. The appropriate identification of the limits follows:

Theorem 2.1. (a) For $0<k<1$

$$
A G_{2}(1, k)=1 /{ }_{2} F_{1}\left(1 / 2,1 / 2 ; 1 ; 1-k^{2}\right) .
$$

(b) For $0<s<1$

$$
A G_{2}(1, s)=1 /{ }_{2} F_{1}\left(1 / 3,2 / 3 ; 1 ; 1-s^{2}\right) .
$$

Proof. Many proofs of (a) are presented in the first chapter of [4]. 
Several of these can be adjusted to verify (b). It is perhaps easiest to establish (b) as follows. First, note that $A G_{3}(x, y)$ is positively homogeneous and invariant under the iteration. Thus, observe that $G(x):=1 / A G_{3}(1 / x)$ satisfies

$$
G(r)=\left(1+2 s^{*}\right) G(s) \text { if }(1+2 r)\left(1+2 s^{*}\right)=3,
$$

that is when

$$
r=\frac{1-s^{*}}{1+2 s^{*}} .
$$

Next, we use either Theorem 1.5 in [4] or the hypergeometric differential equation $[1,4,11]$ to verify that ${ }_{2} F_{1}\left(1 / 3,2 / 3 ; 1 ; 1-s^{3}\right)$ and $1 / A G_{3}(1, s)$ satisfy the same functional equation and agree at 1 . Hence they coincide. Since the underlying differential equation is of second order with algebraic coefficients this identification reduces to an entirely elementary though fairly complicated calculation.

From (2.1) we may easily derive the following products for

$$
F(s):={ }_{2} F_{1}\left(1 / 3,2 / 3 ; 1 ; s^{3}\right),
$$

which is expressible as

$$
F(s)=\sum_{n=0}^{\infty} \frac{(3 n !)}{(n !)^{3}}\left(\frac{s}{3}\right)^{3 n} .
$$

In terms of $F(2.1)$ becomes the promised cubic transformation:

$$
F(s)=(1+2 r) F(r) \text { if }(1+2 r)\left(1+2 s^{*}\right)=3 .
$$

In explicit form this is the lovely cubic equation: for $x \in(0,1)$

$$
{ }_{2} F_{1}\left(\frac{1}{3}, \frac{2}{3} ; 1 ; 1-x^{3}\right)=\frac{3}{1+2 x}{ }_{2} F_{1}\left(\frac{1}{3}, \frac{2}{3} ; 1 ;\left(\frac{1-x}{1+2 x}\right)^{3}\right) .
$$

It also follows that for $s_{0}:=s$ one has

$$
F(s)=\prod_{n=0}^{\infty} \frac{3}{1+2 s_{n}^{*}}=\prod_{n=1}^{\infty}\left(1+2 s_{n}\right) \quad \text { if } s_{n+1}:=\frac{1-s_{n}^{*}}{1+2 s_{n}^{*}} .
$$

These give practical cubically convergent algorithms for $F$ and so for ${ }_{2} F_{1}(1 / 3,2 / 3 ; 1 ; x)$.

Let us recall the following theta function definitions. The special theta functions are defined by

$$
\theta_{3}(q):=\sum_{n=-\infty}^{\infty} q^{n^{2}}, \quad \theta_{4}(q):=\sum_{n=-\infty}^{\infty}(-1)^{n} q^{n^{2}}, \quad \theta_{2}(q):=\sum_{n=-\infty}^{\infty} q^{(n+1 / 2)^{2}}
$$

and satisfy

$$
\theta_{3}^{4}(q)=\theta_{4}^{4}(q)+\theta_{2}^{4}(q) \quad(\text { Jacobi's identity })
$$

We also will use the Lambert series

$$
P(q):=1-24 \sum_{n=1}^{\infty} \frac{n q^{2 n}}{1-q^{2 n}}
$$


and Ramanujan's evaluation $P\left(e^{-\pi}\right)=3 / \pi$ (see $[4,9]$ ). The standard theory is recapitulated as

Theorem 2.2. Let $|q|<1$ be given.

(a) Then

$$
{ }_{2} F_{1}\left(1 / 2,1 / 2 ; 1 ; k^{2}\right)=\theta_{3}^{2}(q) \quad \text { for } k=\frac{\theta_{2}^{2}(q)}{\theta_{3}^{2}(q)} \text { and } k^{*}=\frac{\theta_{4}^{2}(q)}{\theta_{3}^{2}(q)} \text {. }
$$

Moreover, if $q:=e^{-\pi t}$ then $k^{*}(t)=k(1 / t)$, for re $t>0$.

(b)

$$
\theta_{3}^{2}(q)=1+4 \sum_{n=0}^{\infty}(-1)^{n} \frac{q^{2 n+1}}{1-q^{2 n+1}}
$$

and

(c)

$$
\theta_{3}^{4}(q)=\frac{4 P\left(q^{4}\right)-P(q)}{3} .
$$

Proof. These are classical. Proofs of (a) are presented in the second chapter of [4]. In large part this reduces to the parametrization of the AGM via theta functions. Proofs of (b) and (c) are to be found in Chapter 9 of [4]. (In particular, see (9.1.1) and the identity preceding it.)

Before giving our main result we need to introduce the appropriate multidimensional theta functions. First, we sum over the norms $\left(n^{2}-n m+m^{2}\right)$ of integers in $Q(\omega)$, where $\omega$ is the principal cube root of unity:

$$
L(q):=\sum_{n, m=-\infty}^{\infty} q^{n^{2}+n m+m^{2}}
$$

(analogously $\theta_{3}^{2}$ sums over the norms $\left(n^{2}+m^{2}\right)$ of integers in $\left.Q(i)\right)$. We may also write

$$
\begin{aligned}
L(q) & =\theta_{3}(q) \theta_{3}\left(q^{3}\right)+\theta_{2}(q) \theta_{2}\left(q^{3}\right), \\
L\left(q^{4}\right) & =\left[\theta_{3}(q) \theta_{3}\left(q^{3}\right)+\theta_{4}(q) \theta_{4}\left(q^{3}\right)\right] / 2 .
\end{aligned}
$$

For notational convenience we set

$$
a(q):=L(q), \quad b(q):=\left[3 L\left(q^{3}\right)-L(q)\right] / 2, \quad c(q):=\left[L\left(q^{1 / 3}\right)-L(q)\right] / 2 .
$$

Theorem 2.3. Let $|q|<1$ be given.

(a) If $s=c(q) / a(q)$ then $s^{*}=b(q) / a(q)$ and

$$
{ }_{2} F_{1}\left(1 / 3,2 / 3 ; 1 ; s^{3}\right)=a(q) .
$$

Moreover, if $q:=e^{-\pi 2 t / \sqrt{ } 3}$ then $s^{*}(t)=s(1 / t)$, for re $t>0$.

(b)

$$
L(q)=1+6 \sum_{n=0}^{\infty}\left\{\frac{q^{3 n+1}}{1-q^{3 n+1}}-\frac{q^{3 n+2}}{1-q^{3 n+2}}\right\}
$$


and

(c)

$$
L^{2}\left(q^{2}\right)=\frac{3 P\left(q^{3}\right)-P(q)}{2}
$$

Proof. Part (a) goes as follows. By definition

$$
a\left(q^{3}\right)=[a(q)+2 b(q)] / 3 \text { and } c\left(q^{3}\right)=[a(q)-b(q)] / 3 .
$$

We assert that

$$
a(q)^{3}=b(q)^{3}+c(q)^{3} \quad \text { (basic cubic identity). }
$$

Once this has been established, we argue as follows. As $q \rightarrow q^{3}$ we have a parametric (cubically convergent) solution of $A G_{3}$ with the additional property that $a(0)=b(0)=1$. More explicitly,

$$
A G_{3}(a(q), b(q))=A G_{3}\left(a\left(q^{3}\right), b\left(q^{3}\right)\right)=\cdots=A G_{3}(a(0), b(0)) .
$$

Here we have used the facts that $A G_{3}$ is continuous and $|q|<1$. It follows that $A G_{3}(a(q), b(q))=1$. Since $A G_{3}$ is positively homogeneous,

$$
A G_{3}(1, b(q) / a(q))=1 / a(q) .
$$

Moreover the basic cubic identity (2.3) becomes

$$
[b(q) / a(q)]^{*}=c(q) / a(q),
$$

and (a) now follows from Theorem 2.1(b).

To establish (2.3) we resort to some modular function theory. We first use the two factorizations of $L$ given in (2.2) and the one-dimensional theta transform (in $\S 2.3$ of [4]) to deduce that

$$
t a(t)=a(1 / t), \quad t b(t)=c(1 / t), \quad t c(t)=b(1 / t) .
$$

In particular, it follows that

$$
c(t) / a(t)=s(1 / t)
$$

We re-express $b(q)$ as

$$
b(q)=\sum_{n, m=-\infty}^{\infty} \omega^{(n-m)} q^{n^{2}+n m+m^{2}}
$$

and use the multidimensional theta transform as given in [2] to deduce that

$$
c(q)=\sum_{n, m=-\infty}^{\infty} q^{(n+1 / 3)^{2}+(n+1 / 3)(m+1 / 3)+(m+1 / 3)^{2}}
$$

which shows that $c(q)=q^{1 / 3} f(q)$ with $f$ analytic at 0 . (This also follows from the number-theoretic considerations of Remark 2.4(ii).) Thus $c^{3}(q)$ is analytic. On setting $\Delta(t):=s^{3}(t)+s^{3}(1 / t)$ and using (2.4) we have

$$
\Delta(t)=\Delta(1 / t)
$$


while, for trivial reasons,

$$
\Delta(t+i \sqrt{ } 3)=\Delta(t)
$$

We observe from the cubic modular equation (p. 110 of [4]) that $a(q)$ has a single zero in the $\lambda$-fundamental region at $q_{0}=-e^{2 \pi / 3}$ and check that $b\left(q_{0}\right)=c\left(q_{0}\right)$. Thus $\Delta(i t)$ is a modular function for the group generated by the transformations $t \rightarrow 1 / t$ and $t \rightarrow t+i \sqrt{ } 3$. Additionally, $\Delta$ has no poles. This implies that $\Delta$ is bounded and so constant. Letting $t \rightarrow 0$ allows us to identify the constant: $\Delta(t)=1$.

Part (b) is once again classical and can be deduced from the Lambert series for $\theta_{3}(q) \theta_{3}\left(q^{3}\right)$ due to Lorenz given on p. 287 of [4]. Part (c) then follows from exercise $4 \mathrm{~b}$ ) on the same page, which itself follows from a more general identity of Ramanujan's.

Remarks 2.4. (i) Ramanujan gives modular equations of order $2,5,7,11$ for $F$, but not the fundamental cubic equation (see [3, Chapter 21]). Having found the basic modular function $s(t)$, we can verify these identities much as in Theorem 2.3. The two nicest are in our notation:

$$
s(t) s(2 t)+s^{*}(t) s^{*}(2 t)=1 \quad \text { (quadratic) }
$$

and

$$
s(t) s(5 t)+s^{*}(t) s^{*}(5 t)+3\left[s(t) s(5 t) s^{*}(t) s^{*}(5 t)\right]^{1 / 2}=1 \quad \text { (quintic) }
$$

(ii) The Lambert series in (b) is equivalent to $r_{3}(k)=6\left[d_{1}(k)-d_{2}(k)\right]$, where $r_{3}(k)$ counts representations of $k=n^{2}+n m+m^{2}$ including sign and permutation and $d_{j}$ is the number of divisors congruent to $j \bmod 3$.

Also

$$
a(q)=1+\sum_{k=1}^{\infty} r_{3}(k) q^{k}
$$

Elementary arguments show that $r_{3}(3 k)=r_{3}(k)$ and that $r_{3}(3 k+2)=0$ so that

$$
c(q)=q^{1 / 3} \sum_{k=0}^{\infty} \frac{r_{3}(3 k+1)}{2} q^{k}
$$

which again establishes the analyticity of $c^{3}(q)$.

Correspondingly recall that $r_{2}(k)=4\left[d_{1}(k)-d_{3}(k)\right]$, where $r_{2}(k)$ counts representations of $k=n^{2}+m^{2}$ and $d_{j}$ is the number of divisors congruent to $j$ mod 4 (this is the number theoretic equivalent to (b) of Theorem 2.2).

(iii) Both $c^{3}(q)$ and $b^{3}(q)$ have simple Lambert series:

$$
c^{3}(q)=27 \sum_{n=1}^{\infty} \frac{n^{2} q^{n}\left(1-q^{n}\right)}{1-q^{3 n}}, \quad b^{3}(q)=1-9 \sum_{n=1}^{\infty} \frac{\chi_{-3}(n) n^{2} q^{n}}{1-q^{n}}
$$


where $\chi_{-3}(n)$ is $(3 \mid n)$ and

$$
a^{3}(q)=\left[1+6 \sum_{n=1}^{\infty} \frac{q^{n}\left(1-q^{n}\right)}{1-q^{3 n}}\right]^{3} .
$$

(iv) Note that (2.3) is the cubic analogue of Jacobi's identity.

The following logarithmic asymptotes follow from the modularity of $k$ and of $s$ respectively.

Theorem 2.5. (a)

$$
\frac{\pi / 2}{A G_{2}(1, k)} \sim \log \left(\frac{4}{k}\right)
$$

as $k \rightarrow 0^{+}$.

(b)

$$
\frac{\pi / 2}{A G_{3}(1, s)} \sim \frac{3 \sqrt{3}}{4} \log \left(\frac{3}{s}\right)
$$

as $s \rightarrow 0^{+}$.

Proof. (b) Use Theorem 2.3 and Theorem 2.1(b) to write

$$
1 / A G_{3}(1, s(t)) \sim A G_{3}\left(1, s^{*}(t)\right) / A G_{3}(1, s(t))=a(1 / t) / a(t)=t,
$$

where $q=e^{2 \pi t / \sqrt{ } 3}$. But $s \sim c(q) \sim 3 q^{1 / 3}$. Thus $t 2 \pi \sim 3 \sqrt{3} \log (3 / s)$.

(a) is similar and is given in [4].

In (a) the error is of the order $k^{2} \log k$; in (b) the error is of the order $s^{3} \log s$.

We finish this section by giving similar results for another hypergeometric function, ${ }_{2} F_{1}(1 / 4,3 / 4 ; 1 ; x)$, which corresponds to $s:=1 / 4$. We consider the mean iteration that commences with $a_{0}:=a, b_{0}:=b$ and computes

$$
a_{n+1}:=\frac{a_{n}+3 b_{n}}{4}, \quad b_{n+1}:=\sqrt{b_{n}\left(\frac{a_{n}+b_{n}}{2}\right)}
$$

and let the common quadratically attained limit be denoted by $A_{4}(a, b)$.

Theorem 2.6. (a) For $0<h<1$

$$
A_{4}(1, h)=1 /{ }_{2} F_{1}\left(1 / 4,3 / 4 ; 1 ; 1-h^{2}\right)^{2} .
$$

(b) If $|q|<1$ and we set

$$
a(q):=\theta_{3}^{4}(q)+\theta_{2}^{4}(q), \quad b(q):=\theta_{4}^{4}(q), \quad c(q):=2 \theta_{2}^{2}(q) \theta_{3}^{2}(q),
$$

then

$$
{ }_{2} F_{1}^{2}\left(1 / 4,3 / 4 ; 1 ; h^{2}\right)=a(q) \quad \text { for } h=\frac{c(q)}{a(q)} \text { and } h^{*}=\frac{b(q)}{a(q)},
$$

where $h^{* 2}+h^{2}=1$.

Moreover, if $q:=e^{-\pi t / \sqrt{ } 2}$, then $h^{*}(t)=h(1 / t)$, for re $t>0$.

(c)

$$
a\left(q^{2}\right)=2 P\left(q^{2}\right)-P(q)
$$


and

(d)

$$
\frac{\pi / 2}{\sqrt{A_{4}(1, h)}} \sim \frac{1}{\sqrt{2}} \log \left(\frac{8}{h}\right)
$$

as $h \rightarrow 0^{+}$.

Proof. Proposition 5.7 in [4], when expressed in terms of theta functions, is precisely (b). Now one checks (using elementary theta function identities) that $a$ and $b$ provide a parametrization of the iteration in the sense that

$$
a\left(q^{2}\right)=[a(q)+3 b(q)] / 4 \text { and } b\left(q^{2}\right)=\{b(q)[a(q)+b(q)] / 2\}^{1 / 2}
$$

from which we deduce (a). Part (c) is a direct consequence of identities (3.2.20) and (3.2.21) for 4th powers of theta functions, coupled with (3.2.19) in [4]. Now (d) follows either from (a) or from the asymptote for $A G$.

Ramanujan [3] gives the corresponding cubic modular equation as

$$
h(t) h(3 t)+h^{*}(t) h^{*}(3 t)+4\left[h(t) h(3 t) h^{*}(t) h^{*}(3 t)\right]^{1 / 2}=1 .
$$

\section{THREE ITERATIONS FOR $\pi$}

We observe that part (c) of Theorems 2.6, 2.3, and 2.2 may be written as $(N-1) g_{N}(q)=N\left[P\left(q^{N}\right)-1\right]-[P(q)-1]$, where $g_{N}$ is related to the corresponding iteration. On summing this identity,

$$
\sum_{n=0}^{\infty} N^{n} g_{N}\left(q^{N^{n}}\right)=\frac{1-P(q)}{N-1}
$$

since $P(0)=1$. For $q:=e^{-\pi}$ the right-hand side is $(1-\pi / 3) /(N-1)$. Using the exact form of $g_{N}$ gives an iteration for $\pi$ that converges with order $N$.

Sum 3.1 (cubic). For $a_{0}:=1, b_{0}:=[(\sqrt{3}-1) / 2]^{*}$ with $a_{n}$ computed from $A G_{3}$

$$
\pi=\frac{3 A G_{3}^{2}\left(1,\left(\frac{\sqrt{3}-1}{2}\right)^{*}\right)}{1-\sum_{n=0}^{\infty} 3^{n+1}\left(a_{n}^{2}-a_{n+1}^{2}\right)} .
$$

We may truncate the sum after $k+1$ terms and replace $A G_{3}$ by $a_{k+1}$ to obtain an approximation $\pi_{k}$ for $\pi$; alternatively we may re-express this iteration as an iteration for $1 / \pi_{k}$.

Iteration 3.2 (cubic). Let $\alpha_{0}:=1 / 3, s_{0}:=(\sqrt{ } 3-1) / 2$ and

$$
\begin{gathered}
\left(1+2 s_{n}\right)\left(1+2 s_{n-1}^{*}\right)=3 \text { where } s^{3}+s^{* 3}=1, \\
\alpha_{n}:=\left(1+2 s_{n}\right)^{2} \alpha_{n-1}-3^{n-1}\left[\left(1+2 s_{n}\right)^{2}-1\right] .
\end{gathered}
$$

Then $1 / \alpha_{n}$ converges cubically to $\pi$. 
The iteration gives $1,5,21,70, \ldots$ digits correct and more than triples accuracy at each step.

Sum 3.3 (quartic). For $a_{0}:=1, b_{0}:=(12 \sqrt{ } 2-16)^{1 / 4}$ with $a_{n}$ computed from two steps of $A G_{2}$, viz.,

$$
\begin{aligned}
a_{n+1} & :=\frac{a_{n}+b_{n}}{2}, \quad b_{n+1}:=\sqrt[4]{\frac{a_{n} b_{n}^{3}+b_{n} a_{n}^{3}}{2}}, \\
\pi & =\lim _{k \rightarrow \infty} \frac{3 a_{k+1}^{4}}{1-\sum_{n=0}^{k} 4^{n+1}\left(a_{n}^{4}-a_{n+1}^{4}\right)} .
\end{aligned}
$$

We may re-express this iteration as

Iteration 3.4 (quartic). Let $\alpha_{0}:=1 / 3, s_{0}:=\sqrt{2}-1$ and

$$
\begin{gathered}
\left(1+s_{n}\right)\left(1+s_{n-1}^{*}\right)=2 \text { where } s^{4}+s^{* 4}=1, \\
\alpha_{n}:=\left(1+s_{n}\right)^{4} \alpha_{n-1}-4^{n}\left[\left(1+s_{n}\right)^{4}-1\right] / 3 .
\end{gathered}
$$

Then $1 / \alpha_{n}$ converges quartically to $\pi$.

The iteration gives $1,8,41,171, \ldots$ digits correct and more than quadruples accuracy at each step.

Sum 3.5 (quadratic). For $a_{0}:=1, b_{0}:=1 / 3$ with $a_{n}$ computed from $A_{4}$

$$
\pi=\lim _{k \rightarrow \infty} \frac{3 a_{k+1}}{1-\sum_{n=0}^{k} 2^{n}\left(a_{n}-a_{n+1}\right)} .
$$

We may re-express the iteration as

Iteration 3.6 (quadratic). Let $\alpha_{0}:=1 / 3, s_{0}:=1 / 3$ and

$$
\begin{gathered}
\left(1+3 s_{n}\right)\left(1+3 s_{n-1}^{*}\right)=4 \text { where } s^{2}+s^{* 2}=1, \\
\alpha_{n}:=\left(1+3 s_{n}\right) \alpha_{n-1}-2^{n} s_{n} .
\end{gathered}
$$

Then $1 / \alpha_{n}$ converges quadratically to $\pi$.

The iteration gives $1,3,8,19, \ldots$ digits correct and more than doubles accuracy at each step.

These sums and iterations seem well suited for extended precision computation of pi.

Added in proof. 1. David Bailey has computed 98,300 digits of Pi by each of these six algorithms (and several previously discovered methods including the Brendt-Gauss-Salamin algorithm) using one processor of a CRAY Y-MP at NASA Ames. The fastest is Sum 3.3 which took 10.215 CPU seconds.

2. Bruce Brendt (private communication) has informed us that he can establish our key result (2.3) by Ramanujan's methods. Moreover, a version of (2.1) and of Theorem 2.3(a) can be found on page 258 of Ramanujan's Second Notebook. As with most of Ramanujan's results these are given without proof. 


\section{REFERENCES}

1. M. Abramowitz and I. Stegun, Handbook of mathematical functions, Dover, New York, 1964.

2. R. Bellman, A brief introduction to theta functions, Holt, Reinhart and Winston, New York, 1961.

3. B. C. Berndt, Ramanujan's Second Notebook, Springer-Verlag (to appear).

4. J. M. Borwein and P. B. Borwein, Pi and the AGM-A study in analytic number theory and computational complexity, Wiley, 1987.

5. __ Quadratic mean iterations, (monograph in preparation).

6. __ More Ramanujan-type series for $1 / \pi$, Ramanujan Revisited, Academic Press, 1988, pp. 359-374.

7. D. V. Chudnovsky and G. V. Chudnovsky, Approximations and complex multiplication according to Ramanujan, Ramanujan Revisited, Academic Press, 1988, pp. 375-472.

8. G. H. Hardy, Ramanujan, Cambridge Univ. Press, London, 1940.

9. S. Ramanujan, Modular equations and approximations to $\pi$, Quart. J. Math. 45 (1914), 350-372.

10. J. Tannery and J. Molk, Fonctions elliptiques, Vols. 1 and 2, 1893; republished by Chelsea, New York, 1972.

11. E. T. Whittaker and G. N. Watson, $A$ course of modern analysis, 4th ed., Cambridge Univ. Press, London, 1927.

Department of Mathematics, Statistics and Computing Science, Dalhousie UniverSity, HalifaX, Nova Scotia B3H 3J5, Canada 